\title{
Unravelling lifecycles \& physics of radio-loud AGN in the SKA era
}

\section{Anna D. Kapińska ${ }^{* 1,2}$, Martin J. Hardcastle ${ }^{*, 3}$, Carole A. Jackson ${ }^{4}$, Tao An ${ }^{5,6}$, Willem} A. Baan ${ }^{5}$, Matt J. Jarvis $\mathbf{s}^{7,8}$

${ }^{1}$ ARC Centre of Excellence for All-Sky Astrophysics (CAASTRO); ${ }^{2}$ ICRAR, The University of Western Australia, M468, 35 Stirling Hwy, Crawley WA 6009, Australia; ${ }^{3}$ School of Physics, Astronomy and Mathematics, University of Hertfordshire, College Lane, Hatfield AL10 9AB, United Kingdom; ${ }^{4}$ ICRAR, Curtin University, GPO Box U1987, Perth WA 6845, Australia;

5 Shanghai Astronomical Observatory, Chinese Academy of Sciences, 200030 Shanghai, China;

${ }^{6}$ Key Laboratory of Radio Astronomy, Chinese Academy of Sciences, 210008 Nanjing, China;

${ }^{7}$ Oxford Astrophysics, Department of Physics, Keble Road, Oxford OX1 3RH, United Kingdom;

${ }^{8}$ Department of Physics, University of Western Cape, Cape Town 7535, South Africa

E-mail: anna.kapinska at uwa.edu.au

Radio-loud AGN ( $>10^{22} \mathrm{~W} \mathrm{~Hz}^{-1}$ at $1.4 \mathrm{GHz}$ ) will be the dominant bright source population detected with the SKA. The high resolution that the SKA will provide even in wide-area surveys will mean that, for the first time sensitive, multi-frequency total intensity and polarisation imaging of large samples of radio-loud active galactic nuclei (AGN) will become available. The unprecedented sensitivity of the SKA coupled with its wide field of view capabilities will allow identification of objects of the same morphological type (i.e. the entire FR I, low- and highluminosity FR II, disturbed morphology as well as weak radio-emitting AGN populations) up to high redshifts ( $z \sim 4$ and beyond), and at the same stage of their lives, from the youngest CSS/GPS sources to giant and fading (dying) sources, through to those with restarted activity radio galaxies and quasars. Critically, the wide frequency coverage of the SKA will permit analysis of same-epoch rest-frame radio properties, and the sensitivity and resolution will allow full crossidentification with multi-waveband data, further revealing insights into the physical processes driving the evolution of these radio sources. In this chapter of the SKA Science Book we give a summary of the main science drivers in the studies of lifecycles and detailed physics of radio-loud AGN, which include radio and kinetic luminosity functions, AGN feedback, radio-AGN triggering, radio-loud AGN unification and cosmological studies. We discuss the best parameters for the proposed SKA continuum surveys, both all-sky and deep field, in the light of these studies.

Advancing Astrophysics with the Square Kilometre Array

June 8-13, 2014

Giardini Naxos, Sicily, Italy

\footnotetext{
* Speaker.
} 


\section{Introduction}

Large-scale radio surveys compiled over the past 50 years have revealed a multitude of source types that we term 'radio-loud AGN' (Peacock \& Wall 1982; Wall 1994). These objects will be the dominant population of bright sources detected by the SKA. The deep SKA radio surveys will allow detailed and complete analysis of most, if not all, radio-loud AGN populations and provide the basis to resolve some of the most critical questions in this area.

The radio emission from radio-loud AGN is synchrotron emission produced by a population of electrons, transported in a relativistic outflow from the vicinity of the central supermassive black hole, and accelerated to high energies as the jet expands and decelerates to sub-relativistic speeds. This is the dominating source of radio emission from radio-loud AGN down to luminosity densities of $\sim 10^{22} \mathrm{~W} \mathrm{~Hz}^{-1}$ at $1.4 \mathrm{GHz}$; below this luminosity density AGN are referred to as weakly radioemitting and it is under debate whether their radio emission is still dominated by an active nucleus rather than star formation. The subject of weakly radio-emitting AGN is covered elsewhere in this volume (Orienti et al. 2015, see also §6.1). Based on unification models (e.g. Barthel 1989; Urry \& Padovani 1995; Jackson \& Wall 1999), radio-loud AGN are often distinguished as radio galaxies, radio-loud quasars and BL Lac-type objects (dependent on the orientation to the observer). Radio galaxies and radio-loud quasars are further broadly divided into unresolved or compact symmetric objects, FR I and FR II type sources (including peculiar morphologies) and giant radio galaxies (see §2.1; classification of different life stages and radio morphologies). Here, we consider all of these radio-loud AGN, with no selection on multi-wavelength properties of their hosts.

The physics of, and physical conditions in, the radio-emitting plasma are of great interest in themselves, but radio-loud AGN are important for a number of other reasons. Firstly, they provide an obscuration-independent method of selecting AGN out to the highest redshifts, and in some cases, e.g. radiatively inefficient, low accretion-rate AGN radio emission gives us the only method of measuring the output and accretion rate of the system. Secondly, radio-loud AGN are now routinely invoked in models of galaxy formation and evolution, where they provide a socalled 'feedback' mechanism. AGN feedback is now thought to be one of the main mechanisms preventing the cooling of large-scale gas and the consequent growth of the host galaxies (Bower et al. 2006; Croton et al. 2006). Finally, we expect the SKA surveys to reach nJy levels, detecting statistically significant numbers of sources across the wide range of the radio luminosity function (RLF) at all cosmic epochs. When coupled with sufficient angular resolution and multi-waveband data, it will be possible to separate the contribution of radio emission due to an active nucleus from that due to ongoing or bursting star formation (SF; McAlpine et al. 2015).

In this Chapter we primarily focus on how the SKA will reveal the evolution of the radio-loud AGN populations characterised by their radio morphologies and luminosity densities, and at the same time directly provide the necessary radio data for studies of the radio source physics for the first time. There are a number of key questions that these deep samples can address.

- What is the RLF at all cosmic epochs?

There is a huge range of radio AGN luminosity densities; in the local Universe this extends from $10^{22}$ to $10^{27} \mathrm{~W} \mathrm{~Hz}^{-1}$ at $1.4 \mathrm{GHz}$ (Mauch \& Sadler 2007). Due to the Malmquist effect, deep samples are highly biased towards high luminosity sources near to the limiting 
magnitude at each epoch. The result is that RLFs derived from 'complete' small-area radio samples are limited in accuracy and fail to fully probe the breadth of the full RLF. Although it is well established that the RLF evolves steeply for the overall radio-loud AGN population (note the steepness is luminosity dependent, e.g. Dunlop \& Peacock 1990; Sadler et al 2007; Donoso, Best \& Kauffmann 2009; Rigby et al 2011; McAlpine, Jarvis \& Bonfield 2013; Best et al. 2014, among many others), it is still not clear whether it is sources themselves or number of sources that become brighter.

- Is there a link between the evolving radio-loud AGN RLF and the evolution of galaxies and galaxy clusters? Are feedback processes inherent to all radio-loud AGN, or to just a subset of them, and what does this reveal about the physical processes within these populations?

Although interaction between the radio lobes and the hot ambient medium is directly observable in X-rays in the local Universe, it is still an open question whether the physics of radio galaxies is consistent with the role they are thought to play in the models of galaxy formation and evolution (Cattaneo et al 2009; McNamara \& Nulsen 2012). Studies of radio-loud AGN populations in the local Universe (e.g. Best \& Heckman 2012) show that there is a fundamental dichotomy between hosts of high- and low-excitation radio galaxies, and that it is the low luminosity radio sources that drive the AGN activity at $z<0.2$ (e.g. Shabala, Kaviraj, \& Silk 2011). A number of authors attempted to implement AGN feedback into galaxy evolution models (e.g. Shabala \& Alexander 2009), but deep radio-loud AGN samples of wide range of luminosity densities and at $z>0.5$ are required to validate the models.

- What is the kinetic luminosity function (KLF) for AGN?

The radio luminosity density is the detectable signature of a radio-loud AGN, but, as we will discuss in this paper, this bears only a weak relationship to the intrinsic kinetic luminosity (jet power) of AGN. By providing multi-frequency, high-resolution images for large samples of radio-loud AGN, the SKA will give us the best possible chance to break the luminosity density/kinetic power degeneracy and therefore understand the power input by AGN to their host environments and supermassive black hole growth over cosmic time (for population studies see e.g. Kapińska \& Uttley 2013, for a case study see Hardcastle et al 2012).

- What drives the AGN evolution? Is the 'radio-loud AGN' activity a singular phase for a galaxy, either long- or short-duration, or is it cyclic? What triggers the fuelling cycle?

At present we know of a number of radio-loud AGN that show signatures of previous activity episodes (Schoenmakers et al. 2000), but it is still not clear whether all radio sources are retriggered or only some fraction of them (e.g. Saikia \& Jamrozy 2009), or even what triggers radio activity. A number of authors have attempted to tackle this problem via both statistical population as well as case studies at low-redshifts (for recent works see e.g. Shabala et al. 2008; Janssen et al 2012; Kaviraj et al. 2014; Maccagni et al. 2014). However, deep radioloud AGN samples of a wide range of luminosity densities at $z>0.5$ are required to extend these studies to higher redshifts (e.g. Karouzos, Jarvis, \& Bonfield 2014).

In what follows we assume that continued progress in wide and deep optical/IR imaging and spectroscopic surveys will be such that it will be possible to identify a large fraction of the observed 
radio galaxy population and assign redshifts to the sources - a precondition for any study of a population and its physics. Further discussions of radio-loud AGN hosts must involve a discussion of the expected optical survey coverage by the start of SKA science operations - this is discussed elsewhere in this volume (Antoniadis et al. 2015; Bacon et al. 2015; Kitching et al. 2015).

The chapter is composed as follows. In Section 2 we present our current view of the lifecycle of a radio galaxy, and our current observational and theoretical understanding on the radio source evolution as it ages throughout its lifetime. In Sections $3-5$ we separately discuss each stage of a radio-loud AGN life, from a radio galaxy birth, through its mid-life, to its death; in each of those sections we consider the necessary SKA1 and SKA receivers for each of the radio source class (i.e. life phase) observations. In Section 6 we take a broader view on the radio-loud AGN populations, and discuss them in terms of AGN duty cycles, AGN unification and cosmological studies. A brief summary of the SKA elements for this study is given in Section 7. We assume a flat Universe with the Hubble constant of $H_{0}=67 \mathrm{~km} \mathrm{~s}^{-1} \mathrm{Mpc}^{-1}$, and $\Omega_{\mathrm{M}}=0.685$ and $\Omega_{\Lambda}=0.315$ (Planck Collaboration et al. 2013) throughout the paper.

\section{Lifecycles of radio-loud AGN}

The typical timescales of AGN radio activity are estimated to be $\sim 0.1$ Gyr (e.g. Wan, Daly, \& Guerra 2000; Kapińska, Uttley \& Kaiser 2012; Antognini, Bird, \& Martini 2012). Once the radio activity is triggered, the launched jet expands through the host galaxy and ambient medium until the jet supply ceases and the radio source slowly fades radiating away the remaining energy stored in radio lobes. A series of these events is what we refer to as a 'lifecycle' of a radio source.

\subsection{How complete is our current picture?}

The observed populations of radio-loud AGN fall into reasonably well defined classes distinguished by radio morphology, luminosity density and physical size (the latter of which is generally interpreted to be proportional to age). The smallest size radio galaxies, the so-called Compact Symmetric Objects (CSO, < 500 pc; Wilkinson et al. 1994), Gigahertz Peaked Spectrum (GPS, $<1$ kpc with turnover broadband radio spectra; Blake 1970; Stanghellini et al. 1990; O’Dea, Baum \& Stanghellini 1991) and Compact Steep Spectrum (CSS, < 10 kpc; Peacock \& Wall 1982; Fanti et al. 1990; O'Dea 1998) sources are compact radio sources completely embedded in the host galaxy. They are believed to be predominantly young, 'start-up' or 'baby radio-galaxies', approximately $10^{3}-10^{5}$ years old (Section 3 ). These sources may be resolved at VLBI angular resolutions, where they often reveal morphologies similar to those of more extended, of the order of 100-kpc, sources (e.g. Readhead 1995; Snellen, Schilizzi \& van Langevelde 2000). At kiloparsec scales, the Fanaroff-Riley (1974) class I and II (FR I, FR II respectively) are distinguished (Section 4). According to unification models (Urry \& Padovani 1995; Owen, Ledlow \& Keel 1996; Wall \& Jackson 1997; Jackson \& Wall 1999), these can be observed at various angles disguising themselves at times as, for example, core-dominated quasar and blazar sub-populations. FR I and FR II type sources are found to be typically $\sim 10^{7}$ years old (Section 6).

A radio source is thought to evolve through these phases as it ages: from young, compact and luminous CSO/GPS and CSS sources, the jets of which strongly interact with dense interstellar medium (ISM) as they try to leave the host galaxy, to the large-scale FR I and FR II stage 
at which the relativistic jets extend into the inter-galactic (IGM) and intra-cluster medium (ICM). This paradigm was first proposed by Phillips \& Mutel (1982) and Carvalho (1985), and further refined by Readhead (1995), Readhead et al. (1996) and Snellen et al. (1999, 2000), and is based on high resolution observations of young radio sources. With the recent observational advances we can now extend these evolutionary tracks to the very late stages of radio source evolution which include giant (> 1 Mpc; Komberg \& Pashchenko 2009), dying (Parma et al. 2007), and re-started radio galaxies (Schoenmakers et al. 2000, Section 5).

Whilst simple and appealing, this widely accepted evolutionary path may represent just one of many possible evolutionary tracks of radio-loud AGN - perhaps the longest, main lifecycle path. Alternative paths may include sources that do not reach the giant phase stage, the FR I/FR II stage, or even the CSS stage (e.g. Marecki, Spencer \& Kunert 2003). Recent observational evidence for such alternatives comes from the existence of so-called young faders (Kunert-Bajraszewska, Marecki \& Spencer 2004; Kunert-Bajraszewska et al. 2010), a class of compact, low radio luminosity density and small-scale CSS sources that resemble large-scale dying radio galaxies. It is still not clear what causes the radio activity to cease and why it may happen on a wide range of timescales, with some radio sources becoming long-lived giants and others dying in their infancy. For example, in the discussion on why only some FR Is and FR IIs evolve to Mpc scales, longer lifetimes, more powerful engines, or under-dense environments have been suggested as a solution, but no consensus has yet been reached (Machalski \& Jamrozy 2006; Jamrozy et al. 2008; Komberg \& Pashchenko 2009; Kuźmicz \& Jamrozy 2013).

A true over-abundance of a young class of radio sources would advocate the view that some radio activity is indeed terminated prematurely. Our current radio source population counts suffer from well-recognised selection biases. Deep, sensitive and complete $N(z)$ measurements of each, well defined radio source type are required. However, selection criteria for the samples must ensure inclusion of all types of radio source at the same time to allow for the lifecycle analyses. Such deep measurements have also a potential to help us to investigate the weakly radio-emitting source population (weak-radio AGN), analyses of which are pivotal in the investigations of AGN duty cycles (Section 6).

\subsection{Our theoretical understanding of radio-loud AGN evolution}

Building on the seminal work undertaken in the 1970s (Blandford \& Rees 1974; Scheuer 1974), tremendous progress has been made especially in the past 20 years towards an analytical understanding of the physics and evolution of extragalactic radio sources. Semi-analytical approximations developed for classical double FR II radio sources (Kaiser \& Alexander 1997; Kaiser, Dennett-Thorpe \& Alexander 1997; Blundell, Rawlings \& Willott 1999), are being extended to both young GPS/CSS (Snellen et al. 2000; Alexander 2000, 2006; An \& Baan 2012; Maciel \& Alexander 2014) and dying radio galaxy stages (Komissarov \& Gubanov 1994; Kaiser \& Cotter 2002). The latter has been supported by observations of cavities in X-ray brightness maps of galaxy clusters (considered to be signatures of radio source activity; Bîrzan et al. 2004; McNamara $\&$ Nulsen 2007), as well as the discovery of so-called double-double (re-started) radio galaxies (Schoenmakers et al. 2000). The most recent developments include analytical modelling of restarted radio sources (Kaiser, Schoenmakers \& Röttgering 2000; Brocksopp et al. 2011) that can account for multiple activity episodes. Very few radio sources with signatures of re-started radio 


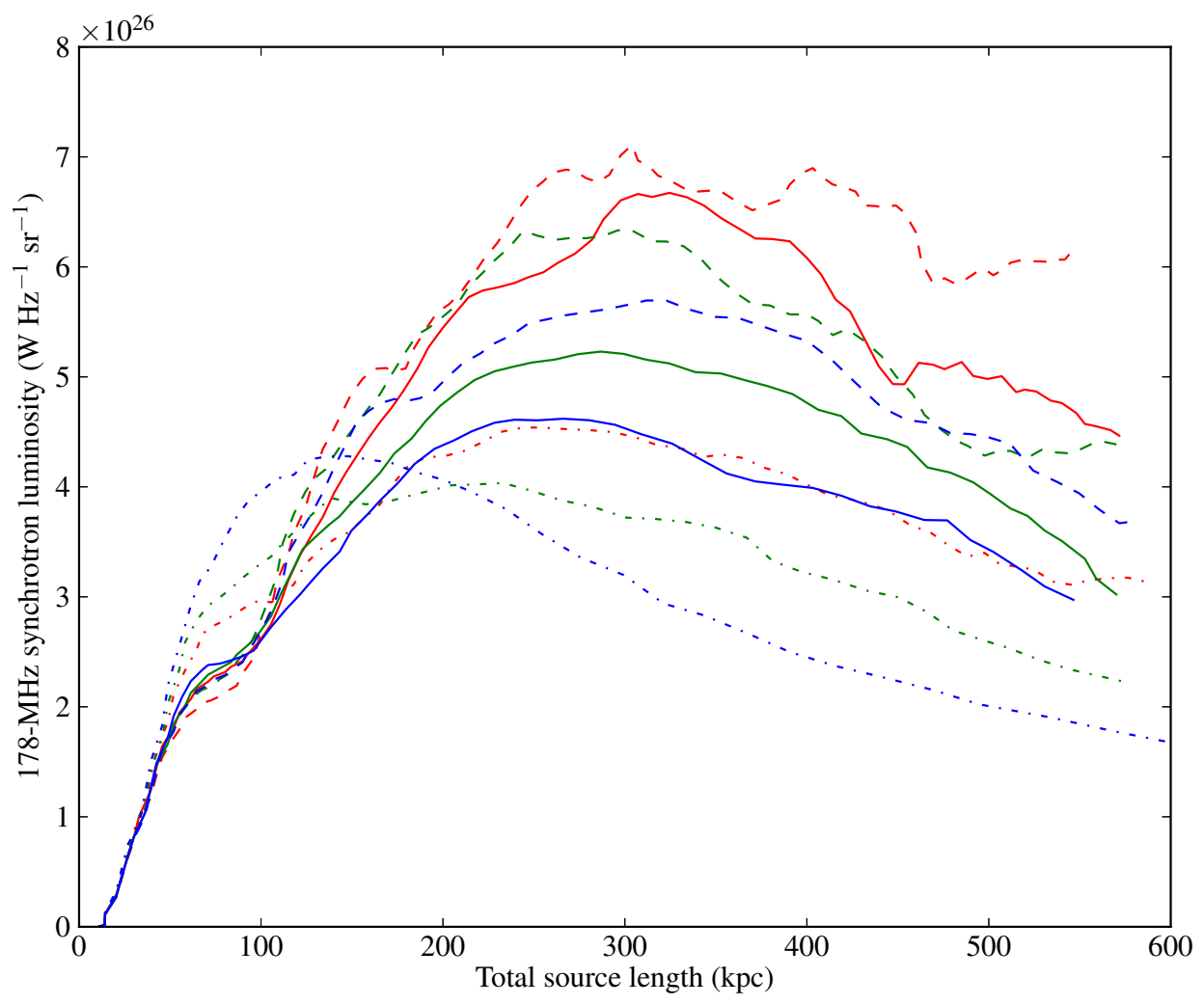

Figure 1: The evolution of radio luminosity density as a function of source physical size as seen in 3D MHD simulations. The different coloured lines show the evolution of a radio source of the same jet power $\left(10^{38} \mathrm{~W}\right)$ in different plausible group/cluster environments, where dot-dashed lines represent poor environments, solid lines intermediate and dashed lines rich environments, and the colours denote the steepness of the density profile in the $\beta$-model (King 1962, blue represent the steepest and red the flattest profile). Note the wide scatter in the luminosity densities at late times and the significant difference between the early luminosity densities and those in the 'plateau' phase at a few hundred kpc. Standard estimates of jet kinetic power (based on scaling relations) would assign a wide range of different kinetic powers to the source simulated here, depending on the part of the lifecycle observed and the environment it inhabits. Figure adapted from Hardcastle \& Krause (2014).

activity have been observed to date - the semi-analytical models are based on fewer than 30 known cases of re-started radio galaxies and on only one source with clear signatures of two episodes of previous activity (Brocksopp et al. 2007, 2011). However, the existence of these sources and understanding of the physics involved is crucial in identifying the radio AGN activity (re)triggering processes and determining duty cycles of radio sources. At the same time, numerical models involving realistic environments are now reaching the stage where they can be used to produce estimates of the evolution of integrated and resolved source properties that complement, and in some cases improve upon, those available from analytic modelling (e.g. Figure 1).

On the other hand, FR I sources are notoriously difficult to model analytically or numerically because of their complex, turbulent and often heavily disturbed radio structures. A number of 
attempts have been made (Laing, Canvin \& Bridle 2003; Luo \& Sadler 2010), but no general analytical model exists that can predict properties of the FR I class as a whole. This is particularly important, as FR Is are thought to be much more numerous at low luminosity densities; it is possible that they will comprise the majority of the radio-loud AGN population at high redshift, low luminosity density radio samples; however, evidence for their high numbers is currently limited to a few studies in deep fields (Simpson et al. 2012; McAlpine, Jarvis \& Bonfield 2013). Numerical approaches to the problem of modelling FR I radio galaxies have been so far focusing predominantly on dynamical evolution of their radio structures (e.g. Perucho \& Martí 2007; Perucho et al. 2014). Sensitive, deep SKA observations that will be available for a large number of such sources are required for further theoretical advances [detailed discussion on this subject is covered elsewhere in this volume; see Agudo et al. (2015); Laing (2015)].

However, analytical models investigating the plausible transition of FR II sources into FR Is have been developed (Wang et al. 2011; Turner \& Shabala 2015), and it is also thought that all radio sources start off with the FR II morphology. Furthermore, we have also discovered a curious class of hybrid radio morphology objects, which show properties of both FR I and FR II sources (Gopal-Krishna \& Wiita 2000; Kapińska et al. 2015). This has direct implications for studies of the AGN host types, environments and their evolution across cosmic time, especially in terms of the observed FR dichotomy (Saripalli 2012), and are already allowing us to statistically model young radio sources, FR IIs and the FR transition populations.

Clearly, observational advances drive our theoretical understanding of radio sources; only by combining both can we study the physical properties of radio-loud AGN that cannot be measured directly (Blundell, Rawlings \& Willott 1999; Kapińska, Uttley \& Kaiser 2012), but are crucial in the studies of galaxy and galaxy clusters evolution, and AGN feedback and activity.

\section{The birth of radio galaxies}

Young, compact radio sources are expected to be very numerous (Fanti et al. 1990), but are generally poorly studied in large samples. The observed complete, flux density limited parent samples often impose significant constraints on the population of radio galaxies that can be detected; most samples are biased towards middle-aged radio sources where the radio luminosity density is expected to be the highest for a given jet kinetic power (Figure 1). There is also a strong bias of GPS/CSS sources to higher redshifts as compared to 100-kpc scale radio galaxies (Snellen et al. 2000). In recent years, a number of faint GPS and CSS samples have been constructed probing lower intrinsic luminosities of these objects (Snellen et al. 1999; Tschager et al. 2003; KunertBajraszewska et al. 2010); but it is important to bear in mind that such samples are often constructed in a very different way than typical, complete samples of extragalactic radio sources, thus making it difficult to compare them to larger, and more evolved radio galaxies.

CSO/GPS and CSS sources are considered to be predominantly young radio galaxies, with typical ages of $10^{3}-10^{5}$ years (Owsianik \& Conway 1998; Murgia et al. 1999; Polatidis \& Conway 2003). However, it is often difficult to distinguish truly young sources from objects whose expansion is 'frustrated' by interaction with a dense ISM (van Breugel, Miley \& Heckman 1984; O’Dea, Baum \& Stanghellini 1991; An \& Baan 2012). There is also increasing evidence that young double radio sources can have a substantial effect on the ISM of their hosts (Croston, Kraft \& Hard- 
castle 2007; Croston et al. 2009; Heesen et al 2014); this seems to be also true for the small radio sources associated with canonically radio-quiet AGN (Mingo et al. 2012).

Estimation of the ratio of young to older, large radio galaxies gives crucial constraints on the lifetime distribution of such objects (i.e. what fraction of them survive to the ages of $10^{7}$ years implied by dynamical and spectral ageing studies of 100-kpc scale radio galaxies), and hence on the accretion history of supermassive black holes that power them. It seems very plausible that there is substantial 'infant mortality' in radio galaxies, i.e. that many do not last long enough to reach the largest sizes and highest luminosity densities (Kunert-Bajraszewska, Marecki \& Spencer 2004; Kunert-Bajraszewska et al. 2010; An \& Baan 2012; Maciel \& Alexander 2014). This has been theoretically discussed by Reynolds \& Begelman (1997), and fading CSS as well as GPS sources with re-started activity have also been observed (Baum et al. 1990; Kunert-Bajraszewska et al. 2010). Deep and complete $N(z)$ measurements are crucial here to answer question whether all these small scale radio galaxies are progenitors of larger-scale FR I and FR II sources and to our overall understanding of the AGN lifecycles and the activity patterns of their central engines.

\subsection{Required SKA elements and the SKA surveys}

With the SKA1 baseline design (Dewdney et al 2013), survey depths will be such that we have a realistic chance of detecting the small-scale counterparts of all radio galaxies of even the lowest jet kinetic power ( $10^{35} \mathrm{~W}$; Hardcastle, Evans \& Croston 2006) out to $z \sim 0.6$ using all-sky surveys with SKA1-SUR or SKA1-MID (Band 2, $1.4 \mathrm{GHz}$ ) and deep field observations with high frequency receivers on SKA1-MID (Band 4 and 5, $4 \mathrm{GHz}$ and $9.2 \mathrm{GHz}$ respectively). Assuming the jet kinetic power - luminosity density scaling relation of Willott et al. (1999) holds, and scaling down the resulting luminosity density by a few orders of magnitude to account for evolution during the radio source growth (Figure 1) we estimate luminosity densities of $\sim 10^{22} \mathrm{~W} \mathrm{~Hz}^{-1}$ for young radio sources which could be progenitors of the weakest FR II radio galaxies. Given the numbers of FR IIs, we would expect at least of order one of these young sources per square degree on the sky, and possibly many more if many FR II-power jets turn off before they reach 100-kpc scales.

The main limitation of SKA1 for the study of CSS/GPS sources will be angular resolution; if we assume 0.05 arcsec resolution at the highest frequencies (SKA1-MID Band 5), then at $z=0.3$ we will resolve only sources with linear sizes $>0.66 \mathrm{kpc}$, and $>1.35 \mathrm{kpc}$ at higher redshifts (assuming source size at least $3 \times$ the beam size). Lower frequencies (and especially SKA1-LOW) will not be useful for detailed radio morphology analyses. However, the high frequency capabilities will still allow an almost complete survey of the whole of the low- and mid- $z$ radio galaxy population down to the smallest sizes and lowest powers. Using a combination of angular resolution and in-band spectral information we will be able to distinguish young objects (steep spectrum, double lobe structure) from beamed, core-dominated systems (flat spectrum). The broad-band spectral coverage of SKA1-MID, -SUR and -LOW, especially when combined with each other, is crucial for selection of the GPS sources and investigation of their physics (Callingham et al. 2015). Early science can be carried out by aiming to be complete to some less ambitious combination of jet kinetic power lower limit and redshift upper limit, which will still give valuable insights into the properties of the lower-power sources.

All of these observations will provide a large number of sources too small for the SKA1 to resolve but which can be followed up by longer-baseline instruments such as the EVN, which 


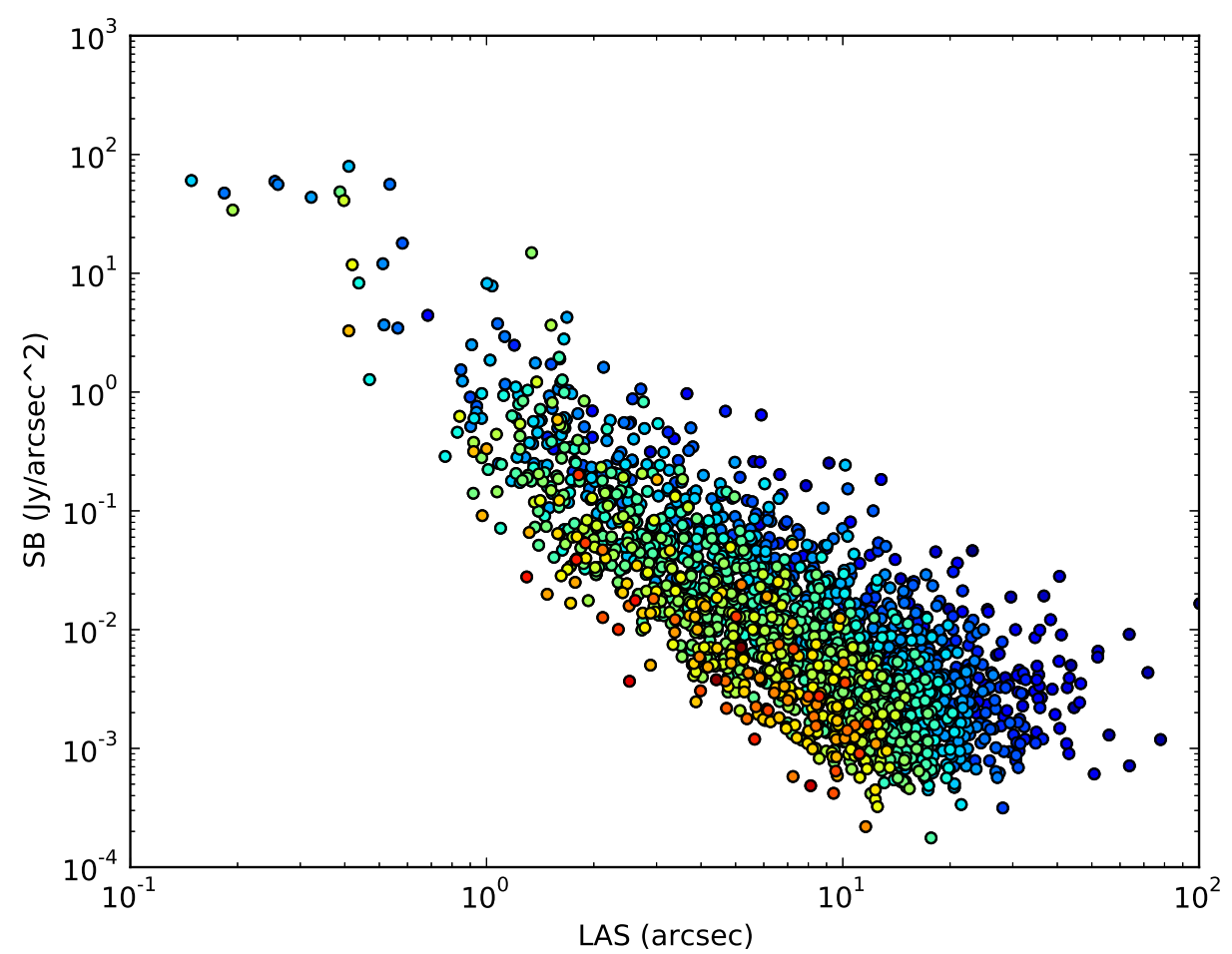

Figure 2: Largest angular size (LAS) and $151 \mathrm{MHz}$ surface brightness (SB) for FR II radio galaxies from the SKADS SEX simulations (Wilman et al. 2008). Colour coding indicates redshift, from dark blue $(z=0)$ to $\operatorname{red}(z \simeq 6)$.

are expected to provide an important complementary facility in the SKA era, unless the SKA VLBI facility is incorporated from the beginning of the telescope operations. Furthermore, detailed polarimetric studies of individual objects remain crucial to both understanding the relativistic jetISM interaction on parsec scales (An et al. 2010; Agudo et al. 2015; Johnston-Hollitt et al. 2015), and establishing the fraction of young vs. frustrated CSO/GPS and CSS sources.

In the final SKA stage we would expect to be able to see essentially every source with jet kinetic power $>10^{35} \mathrm{~W}$, independent of its age except for sources less than a few hundred years old, out to $z=0.5-1.0$ (Bands 2 and 5), and all FR II-power ( $>10^{36} \mathrm{~W}$; Rawlings \& Saunders 1991) start-up galaxies out to $z \sim 3$, well into the regime where cosmological evolution of the radio source population becomes important. Assuming the angular resolution will be improved to at least 0.005 arcsec, we will be able to resolve sources of linear sizes $>150 \mathrm{pc}$ at all redshifts.

\section{Radio galaxies in their mid-life: evolution, jet power and environmental impact}

\subsection{Detailed physics of jets and radio lobes}

Large radio galaxies, with physical sizes of tens to hundreds of $\mathrm{kpc}$, are the best-studied class of radio galaxy and have the best-understood effects on their ambient medium - the hot phase of the IGM/ICM; these are the sources generally thought to be responsible for the 'radio-mode feedback' 
that prevents the hot phase from cooling out onto the most massive galaxies (Bîrzan et al. 2004; McNamara \& Nulsen 2007). As shown in Figure 1, the brightness of the radio emission is expected to peak in this phase of a source's evolution, so these are the objects predominantly selected in large-scale flux density-limited surveys, and will be the easiest to detect and image with the SKA.

Traditionally, radio galaxies detected in surveys have been characterized on the basis of an integrated flux density, and thus an estimate of radio luminosity density - this can be directly used to construct RLFs of radio-loud AGN. However, for AGN feedback or radio source studies what one really wants to know is the jet kinetic power - the instantaneous or time-averaged rate at which radio-loud AGN are transferring energy to their environments. The relationship between the observed radio emission and the jet kinetic power is a long-standing problem (Rawlings \& Saunders 1991; Willott et al. 1999; Kapińska, Uttley \& Kaiser 2012, see also Section 2.2) and it is increasingly clear that the answer is not expected to be simple. At low radio luminosities, a substantial scatter in the relation between radio luminosity density and jet kinetic power is observed (Cavagnolo et al. 2010; Godfrey \& Shabala 2013); this is expected on theoretical grounds, since the radio luminosity density should be a function of the jet kinetic power, the age of the source (Kaiser, Dennett-Thorpe \& Alexander 1997), radio morphology, and, crucially, the source's environment (Barthel \& Arnaud 1996; Hardcastle \& Krause 2013) which we know to differ widely from source to source even at a fixed redshift (Ineson et al. 2013). In the case of weakly radio-emitting AGN, we can assess the interaction with their host environments just by measuring a luminosity density in some band and applying a bolometric correction. The problems described above mean that, for radio-loud AGN, no comparable correction exists.

The jet kinetic power-environment-age degeneracy can be broken with observations that characterize not just the radio luminosity density but also the physical size, aspect ratio and spectral age of the source; in principle, this can allow not just the jet kinetic power but also the properties of the environment (possibly including an estimate of the heating rate) to be determined directly from radio observations. Spectral age measurements can be made by fitting to the spatially resolved broad-band spectrum of the source (Harwood et al. 2013), and so to apply these techniques we need high spatial resolution (to image the lobes) as well as high spatial dynamic range (so that the largest scales are also well mapped). For this method to result in robust measurements, large samples of the order of hundred - thousand sources are required.

\subsection{The role of SKA}

To illustrate the potential of the SKA1 baseline design (Dewdney et al 2013) in this area we consider the FR II radio sources in the SKADS simulations of the extragalactic sky (Wilman et al. 2008). These simulations are probably not accurate enough to give reliable estimates for the properties of the young source population discussed in Section 3, but should be adequate to consider the well-resolved FR II population. We focus on the FR IIs here since the dynamics and particle content of these 'classical double' sources are (relatively) well understood (Kaiser \& Alexander 1997; Celotti 2003). Figure 2 shows the mean $151 \mathrm{MHz}$ surface brightness of all FR IIs in the simulation as a function of source largest angular size; their surface brightness at $5 \mathrm{GHz}$ is expected to be a factor 6 to 30 lower. With 0.05 arcsec beam (SKA1-MID Band 5) we can achieve modest levels of resolution (at least 5 beams across these large-scale sources) for most FR IIs in the simulation, and do a lot better for the bulk of the population. While the $\mu \mathrm{Jy}$ sensitivity and 


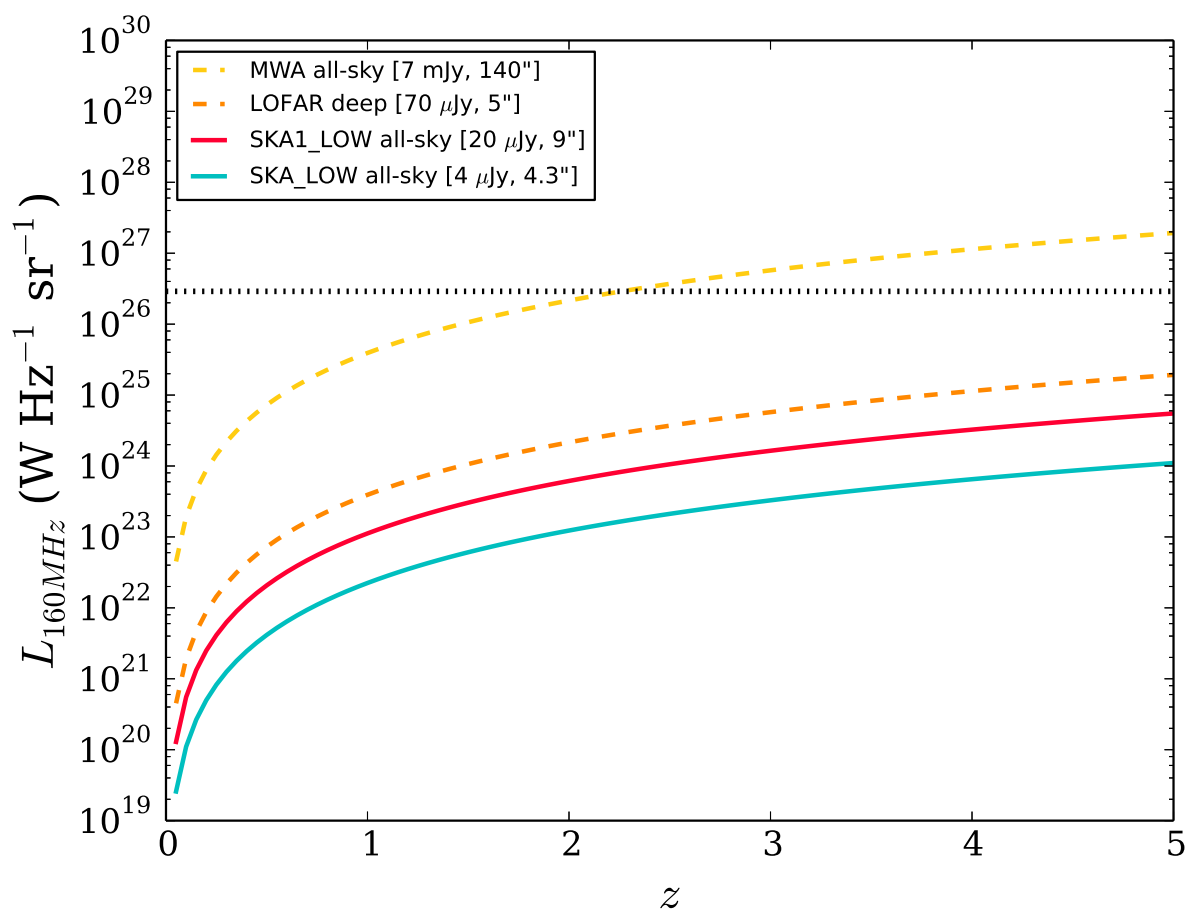

Figure 3: $L-z$ limits of planned SKA1-LOW and SKA-LOW continuum all-sky surveys (solid lines; deep field observations are not feasible, confusion limited). We assume SKA-LOW will reach $90 \mathrm{~km}$ baselines (arm-core, $2 \times$ SKA1-LOW) and angular resolution of 4.3 arcsec. For comparison, the current SKA-LOW pathfinders and precursors are plotted (dashed lines): the ongoing MWA all-sky survey ( $7 \mathrm{mJy} \mathrm{rms}$ ) and deep LOFAR Surveys observations (expected $70 \mu \mathrm{Jy}$ rms for Tier 1). The horizontal line (dotted, black) marks the traditional FR I/FR II divide (Fanaroff \& Riley 1974) scaled to $160 \mathrm{MHz}$ (assuming $S \propto v^{-0.75}$ ).

exceptional $u v$-coverage of the projected surveys will allow the typical surface brightness of all but the faintest lobes to be imaged. Although at lower resolution, the SKA1-SUR in Band 2 (1.4 GHz) will be also very useful for this application as it will provide us with large, wide-field surveys of FR Is and FR IIs compiled within only 2 years on-sky time (Figure 4); we will still be able to resolve over half a population of these sources.

Good constraints on spectral age require broad-band measurements, ideally including lowfrequency observations which would measure the un-aged energy spectrum of the electrons, the so-called injection index, which is known to vary significantly across the source population (Konar \& Hardcastle 2013). The reference design for SKA1-LOW (Dewdney et al 2013), with a beam at best a few arcsec, will not resolve the bulk of these sources, complicating the experiment. However, samples constructed from such low-frequency observations would be the base samples in our analyses of the AGN lifecycles and RLFs as they present the best probe of observing radio-loud AGN unbiased by the effects of relativistic beaming and orientation. At $\mathrm{MHz}$ frequencies, observations preferentially select jet and lobe emission due to its inherent injection spectral signature (i.e. very steeply rising spectra towards lower radio frequencies). The SKA1-LOW all-sky survey, completable within two years on-sky time, is expected to reach rms noise levels of $20 \mu \mathrm{Jy}$ (confusion limited) at angular resolution of $\sim 9 "$. This translates to luminosity densities of $5.7 \times 10^{23}$ $\mathrm{W} \mathrm{Hz}^{-1}$ at $z=2$ (Figure 3) allowing us to detect the vast majority of CSS, FR I and FR II sub- 
populations out to very high redshifts ( $z \sim 4-5$ and beyond), but only at a modest spatial resolution of $20-75 \mathrm{kpc}$ depending on the redshift.

While the SKA1 reference design (Dewdney et al 2013) will allow many thousands of FR II sources to be imaged to high redshift at high-to-moderate resolution, providing an essential testbed for this technique, the final stage of the SKA will be necessary to obtain well-resolved lowfrequency images; this would require baselines of $>300 \mathrm{~km}$. Such baselines will allow us to resolve ( $3-5 \times$ beam size of 1.3 arcsec) over half of the population of FR IIs at these low radio frequencies (Figure 2). This would also extend the study to the much fainter, high-redshift FR I population, and thus allow full analysis of the FR dichotomy at all epochs. Ideally, one would like to reach angular resolutions of 0.4 arcsec as this would allow us to resolve almost whole FR II population; this however, would require baselines as long as $\sim 1000 \mathrm{~km}$ for SKA-LOW.

\section{Death, relics and activity re-triggering}

\subsection{The population of relic radio galaxies}

The prominent features of radio sources (core, jets, hotspots) are fed by the continuous supply of energy from the active nucleus; once the jet activity stops, these features will disappear relatively quickly, and the lobe plasma will continue to expand and to cool via synchrotron and inverse-Compton losses, leaving a 'relic' radio galaxy (Cordey 1987). During this fading phase very strong spectral evolution of the source occurs, with the high radio frequency part of the spectrum developing an ultra steep, exponential cut-off, and the spectral break shifting to lower radio frequencies. Although every galaxy must go through this stage, only a handful examples of true dying radio sources is currently known (Parma et al. 2007; Dwarakanath \& Kale 2009). Reasons for the rarity of such sources may be their low surface brightness and relatively short time they spend in the fading phase as compared to the average lifetime of a radio source; at $\mathrm{GHz}$ frequencies a source will fade away within $10^{4}-10^{5}$ years, while at $\mathrm{MHz}$ frequencies this may take $\sim 10^{7}$ years. Identification of genuinely dying radio galaxies will give important information about lifetimes and duty cycles of extragalactic radio sources. Fading radio galaxies have also implications for AGN feedback since large amounts of the energy supplied by the jet remains stored in the lobes at the end of the active jet phase, and it remains an open question whether, and on what time and spatial scales, that energy is imparted to the ICM.

As discussed in Section 2.1 the cessation of the jet energy supply seems to happen at any stage of radio source growth, and so we need to be searching for dying radio galaxies at all spatial scales, from pc to Mpc scales. Luminosity densities of lobes of fading radio galaxies are $0.3-40 \times 10^{23}$ $\mathrm{W} \mathrm{Hz}^{-1}$ at $1.4 \mathrm{GHz}$ (Parma et al. 2007; Dwarakanath \& Kale 2009), while those of low-luminosity CSS sources, a significant number of which is believed to be in fading phase, to reach as low as $2 \times 10^{23} \mathrm{~W} \mathrm{~Hz}^{-1}$ at $1.4 \mathrm{GHz}$ (Kunert-Bajraszewska et al. 2010). With the currently available allsky surveys (e.g. FIRST, NVSS; Becker, White \& Helfand 1995; Condon et al. 1998) we are able to probe only the 'tip of the iceberg' of this population, at relatively low redshifts $\left(>6 \times 10^{23} \mathrm{~W} \mathrm{~Hz}^{-1}\right.$ up to $z \sim 0.3$ at $1.4 \mathrm{GHz}$ ) assuming the sources are not resolved out.

This is the biggest problem we are presently struggling with - very few instruments can detect such low surface brightness sources, radio structures of which are often spread over multiple 


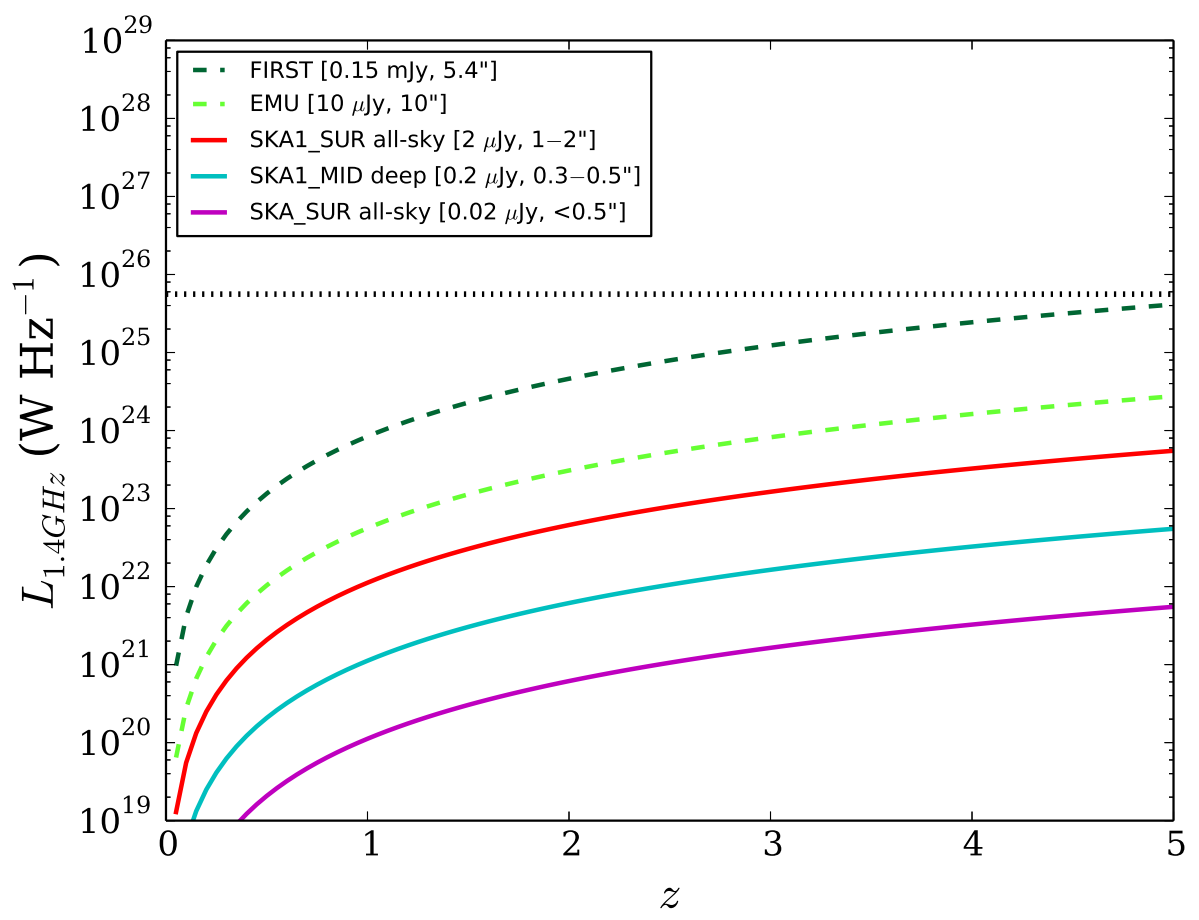

Figure 4: $L-z$ limits of planned SKA1-SUR and SKA1-MID (Band 2) continuum all-sky and deep surveys. We assume SKA-SUR will reach $10 \times$ the sensitivity of SKA1-MID. For comparison, the FIRST survey that have been serving us well over the past 20 years $(0.15 \mathrm{mJy}$ rms at $1.4 \mathrm{GHz})$ and the planned ASKAP-EMU all-sky survey (anticipated $10 \mu \mathrm{Jy}$ ) are plotted (dashed lines). The horizontal line (dotted, black) marks the traditional FR I/FR II divide (Fanaroff \& Riley 1974) scaled to $1.4 \mathrm{GHz}$ (assuming $S \propto v^{-0.75}$ ).

telescope's beams. One of the most spectacular recent examples of hidden imprints of previous activity (fading lobes) is 3C 452, which up to now was believed to be a classical FR II radio galaxy (Sirothia, Gopal-Krishna \& Wiita 2013). How many radio sources have previous activity signatures hidden in such a way? Clearly, there is a hidden world of secret lives of radio-loud AGN we are just starting to uncover. Presently advances and new discoveries are being already made with the existing and new facilities such as Murchison Wide-field Array (MWA; e.g. Hurley-Walker et al. 2014), Giant Metrewave Radio Telescope (GMRT) and Low Frequency Array (LOFAR).

\subsection{What will SKA see?}

As an EoR driven instrument, SKA1-LOW will have tremendous surface brightness sensitivity capabilities, and so will be well adapted to searches for large, faint objects with little compact structure, although it may be difficult to identify them with their host galaxies. In principle, the number of large-scale fading radio galaxies should be comparable to the number of 'alive' large-scale FR Is and FR IIs, but the fading radio galaxies will have much steeper spectra and considerably lower radio luminosity density; surface brightness sensitivity is one of the crucial aspects of the new radio telescopes if we want to obtain large samples of this rare class of radio source.

To demonstrate capabilities of the SKA and its precursors, let us assume a fading radio source of an observed luminosity density $6 \times 10^{23} \mathrm{~W} \mathrm{~Hz}^{-1}$ at $160 \mathrm{MHz}$ and $500 \mathrm{kpc}$ total physical size. This luminosity density and physical size can be easily translated to a brightness temperature per 


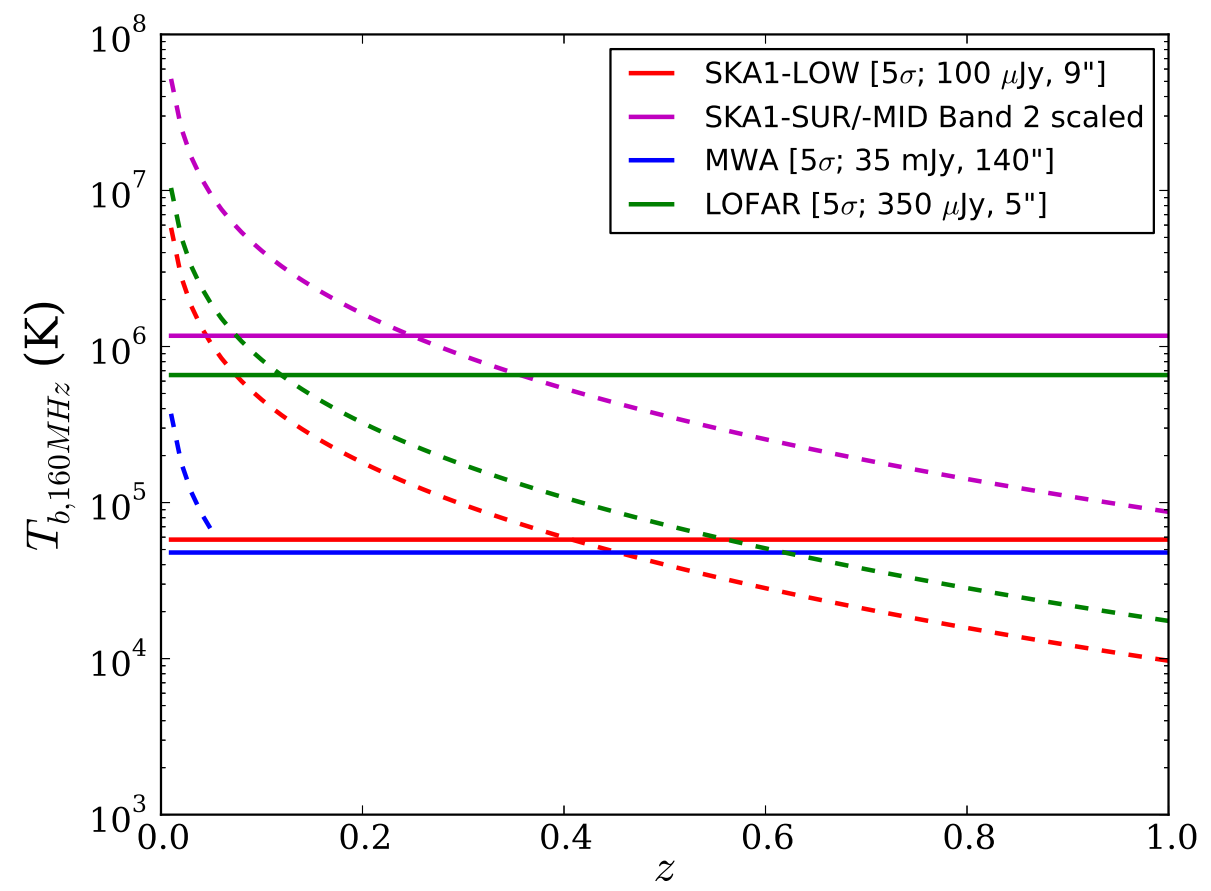

Figure 5: Brightness temperature capabilities of SKA1-LOW and its precursors (MWA, LOFAR) for detecting fading radio source of $500 \mathrm{kpc}$ physical size and total luminosity density $6 \times 10^{23} \mathrm{~W} \mathrm{~Hz}^{-1}$ at $160 \mathrm{MHz}$. Solid lines indicate the brightness temperature sensitivity of each instrument for a given set of flux density limit and angular resolution; $5 \sigma$ values are plotted. Dashed lines indicate the observed surface brightness temperature per beam of the assumed source, as a function of $z$, and are drawn only to a point where the angular size of the source is $>3 \times$ the beam size of the instrument with which we want to detect the source. Both solid and dashed lines of the same colour refer to the same instrument. For a comparison, capabilities of SKA1-SUR/SKA1-MID Band $2(1.4 \mathrm{GHz})$ in detecting such a source are plotted; predicted Band 2 all-sky survey sensitivity of $1 \mu \mathrm{Jy}$ has been scaled to $160 \mathrm{MHz}$ using an assumed steep spectral index of a fading radio source, $\alpha \sim 1$.8. For discussion see Section 5.2. Note that the surface brightness sensitivity of an instrument depends on both its sensitivity (flux density limit) and angular resolution.

telescope's beam and an angular size once a redshift is assigned. As shown in Figure 5, SKA1LOW will be invaluable in searches for such radio sources, being able to detect and resolve them (at least with the modest $3 \times$ beam size) up to $z \sim 0.4$. Furthermore, we attempted to verify capabilities of higher frequency receivers of SKA for this application. Assuming a steep spectral index of the considered fading radio source, $\alpha \sim 1.8$, its total luminosity density at $1.4 \mathrm{GHz}$ would be $1.3 \times 10^{22}$ $\mathrm{W} \mathrm{Hz}^{-1}$ spread over many telescope's beams, but the high sensitivity of SKA1-SUR/-MID will still be able to detect such a source out to $z \sim 0.2$. Those higher frequency receivers will be able to detect less extreme sources at higher redshifts; for instance, a fading radio galaxy of a total luminosity density $6 \times 10^{24} \mathrm{~W} \mathrm{~Hz}^{-1}$ at $1.4 \mathrm{GHz}$ will be detectable out to $z \sim 1.6$, and although SKA1-LOW will detect sources of such luminosity density at $160 \mathrm{MHz} z \sim 2$ and beyond, the former receivers will provide us with much higher resolution. A combination of both arrays may provide us with versatile range of images of dying radio sources. 


\section{Evolution of the AGN population, radio-loud AGN unification and cosmology}

\subsection{Duty cycles of radio activity}

Duty cycles, the relative times a radio source spends in its active and quiescent phases, is a crucial piece of information in our understanding of AGN lifecycles and their impact on the evolving Universe. To constrain these, we need to establish what causes radio sources to shut off and re-trigger, and whether all radio sources go through the re-triggering phase, or only a fraction of them. We need to construct representative RLFs not only of the radio-loud AGN in the midst of their activity (Sections 3-5), but also of those in quiescence that are composed of the weakly radio-emitting AGN; these samples need to be well defined in terms of black hole accretion levels (e.g. Falcke, Körding \& Nagar 2004; Merloni \& Heinz 2008).

It remains an open question as to whether there are underlying physical distinctions between the radio-loud/weak-radio AGN populations, or if there exists a continuum of radio activity which extends to the nuclear radio-quiescent galaxies, dominated by active or evolved star formation (see e.g. Broderick \& Fender 2011; Kimball et al 2011; Mahony et al. 2012, for recent analyses). The sensitive SKA continuum surveys will, for the first time, provide us with large samples of the largely unexplored weakly radio-emitting AGN $\left(10^{20}-10^{22} \mathrm{~W} \mathrm{~Hz}^{-1}\right.$ at $\left.1.4 \mathrm{GHz}\right)$. During the SKA1 stage, with SKA1-SUR/SKA-MID (Band 2) all-sky surveys we will be able to detect the weak-radio AGN population down to luminosity densities of $5 \times 10^{21} \mathrm{~W} \mathrm{~Hz}^{-1}$ (5 $\sigma$ detection) at $z=0.5$. With deep SKA1-SUR/SKA1-MID (Band 2) and SKA1-MID (Band 5) surveys we will go much deeper reaching $1 \times 10^{21} \mathrm{~W} \mathrm{~Hz}^{-1}$ at $z=0.5$ and $4 \times 10^{19} \mathrm{~W} \mathrm{~Hz}^{-1}$ in the local Universe $(z \sim 0.1)$. SKA1-MID Band 5 receivers will also allow us to resolve these sources on linear scales of $0.3 \mathrm{kpc}$ at $z \sim 0.5$. For detailed discussion on the physics of the weak-radio AGN and observations during the SKA era see Orienti et al. (2015).

\subsection{Radio-loud AGN unification}

The deep SKA surveys will sample all radio-loud AGN populations (Section 1) with a subset of these being resolved in suitable detail to model their detailed internal physical processes. However, another view of the radio-loud AGN lifecycles is to view them in terms of populations that manifest themselves as quasars, radio galaxies, and blazars, depending on their orientation on the sky. Clearly, the detailed studies of these sources as described in this paper will improve our view of the radio-loud AGN populations and provide direct tests of simple unification models which extrapolate sparse RLF information with evolutionary scenarios to fit deep source count data (e.g. Owen, Ledlow \& Keel 1996; Wall \& Jackson 1997; Jackson \& Wall 1999). As discussed in Section 2.2 we model AGN lifecycles assuming that FR Is and FR IIs are physically distinct classes of radio source. This view is supported by simple unified models for radio-loud AGN where the FR I and FR II populations are the 'parent' populations of many other observed classes of sources (e.g. Urry \& Padovani 1995; Jackson \& Wall 1999). In adopting these unified models, we can probe both the gross evolution of these populations and disentangle the effects of the AGN lifecycle, as well as map the contribution from weakly radio-emitting AGN and SF sources at least up to $z \sim 0.5$. Angular resolutions of the order of arcsec are sufficient for these studies (e.g. McAlpine, Jarvis \& Bonfield 2013). 
Whilst testing the radio-loud AGN unification models we will obtain a simplified method to map the evolution of the radio-loud AGN population by exploiting the deep source counts, radio luminosity functions and populations under test. These analyses are best done from samples and source counts at low frequencies $(<800 \mathrm{MHz})$; at these frequencies the sources are dominantly steep-spectrum and uncontaminated by relativistic beaming effects. For instance, following the methodology of e.g. Jackson \& Wall (1999) or McAlpine, Jarvis \& Bonfield (2013) we will be able to determine the evolution scenarios that fit best to the observed complete samples. These analyses may be used to provide insight into AGN duty cycles (Section 6.1), and the prevalence of certain types of sources such as GPS/CSS and dying radio galaxies. Using a range of deep counts at a range of observed frequencies $(100 \mathrm{MHz}-$ few $\mathrm{GHz})$ we can test evolutionary and lifecycle scenarios as well as explore the radio-loud / radio-quiet AGN divide.

\subsection{The SKA era precision cosmology}

One of the important aspects of the science described here is that we will identify different populations of AGN, and as presented in Ferramacho et al. (2014) who showed that if one can separate out the various populations, which in turn sample the underlying dark-matter density distribution with a different bias, then the effects of cosmic variance may be overcome in determining the angular power spectrum. This is critical for studying the Universe on the largest scale (Camera et al. 2015). Powerful radio-loud AGN, provide a unique sampling of the underlying dark matter distribution as they are, generally, the most highly biased tracers of the density field, and are detected up to the highest redshifts $(z \sim 6)$. Therefore, when combined with less biased tracers, e.g. star-forming galaxies (Jarvis et al. 2015), they may provide a unique way to understand the largest scales in the Universe, given that the ability to overcome the cosmic variance is dependent on the difference between the bias of the two populations under consideration. The key issue here is to separate AGN from star-forming galaxies, and the high-resolution of SKA1-MID can enable the separation of AGN and star-forming galaxies on either morphology for jet sources $(\gtrsim 0.5$ arc$\mathrm{sec}$ ) or through pure brightness temperature measurements. Furthermore, knowledge of the $N(z)$ is also crucial and the detailed follow-up that would be carried out for this science case, in particular obtaining redshifts, will also be valuable for cosmological science.

\section{Concluding remarks}

A combination of the SKA arrays and their receivers at a wide range of frequencies and angular resolutions are necessary to address the science case discussed in this chapter. In particular, the SKA1-LOW array with its $300 \mathrm{MHz}$ bandwidth, which we assumed here to be centered on $160 \mathrm{MHz}$, is an indispensable tool for searching for old, dying radio sources, and for the construction of complete, flux density and volume limited samples of radio-loud AGN unbiased by the effects of relativistic beaming. SKA1-SUR and SKA1-MID Band 2 (centered on 1.4 GHz, with $800 \mathrm{MHz}$ bandwidth) will provide us with deep, wide-field surveys at a reasonable resolution of $1-2$ arcsec. This will allow for morphological identification of large radio-loud AGN samples, as well as it will provide us with large weak-radio AGN samples in the local Universe. SKA1-MID Band 2, with angular resolution $3-5 \times$ better $(0.3-0.5 \operatorname{arcsec})$, can be used as a follow up for a number of deep fields. Finally, SKA1-MID with its Band 4 and Band 5 (centered on $4 \mathrm{GHz}$ and 
9.2 GHz, with $2.4 \mathrm{GHz}$ and $2 \times 2.5 \mathrm{GHz}$ bandwidths respectively) is particularly useful for high resolution ( 0.05 arcsec) imaging of jets and lobes of both young and evolved radio galaxies for detailed physics analyses. It will be also useful for distinguishing sources within our radio samples that are truly relativistically beamed. The SKA-VLBI facility, if incorporated from the beginning of the telescope operations, will be invaluable for detailed physics investigations of most of radio-loud AGN.

A combination of these SKA arrays and frequency bands will provide us with broad-band radio spectra of the sources - this is crucial for selecting certain types of radio source; e.g. GPS sources are distinguished by their turn-over spectra, while dying radio galaxies are extreme steep spectrum sources $(\alpha>1.8)$. Furthermore, such broad-band spectra will allow for spectral age estimates of the radio sources so important for the radio-loud AGN physics and lifecycle studies.

With such rich data sets we will be able to not only to investigate radio-loud AGN and their engine and model radio source detailed physics, but also to trace the AGN activity (triggering and feedback) up to the high- $z$ Universe and advance the galaxy formation and evolution models. Progress in these crucial science areas is currently hindered by the lack of wide-field, deep radioloud AGN samples that extend to high- $z$, and so is limited only to the local Universe.

Advances are now being made with the SKA pathfinders and precursors, such as MWA, LOFAR, MeerKAT and ASKAP. SKA1 will be much faster than any of these precursors and pathfinders, completing all-sky surveys ( $3 \pi$ sr) within only 2 years on-sky time. It is, however, the full SKA that may revolutionise our understanding of the radio-loud AGN lifecycles and physics, by reaching the unexplored flux density depths of the radio sky.

\section{Acknowledgements}

ADK acknowledges financial support from the Australian Research Council Centre of Excellence for All-sky Astrophysics (CAASTRO), through project number CE110001020. CAJ is a West Australian Premier's Fellow and acknowledges support from the WA Government Department of Premier and Cabinet and from the Curtin University. TA acknowledges financial support from the China Ministry of Science and Technology (grant no. 2013CB837900). The authors thank the referee for detailed comments on the manuscript which improved completeness of this chapter.

\section{References}

Agudo I. et al., 2015, 'Studies of Relativistic Jets in Active Galactic Nuclei with SKA', in proceedings of 'Advancing Astrophysics with the Square Kilometre Array', PoS(AASKA14)093

Alexander P., 2000, MNRAS, 319, 8

Alexander P., 2006, MNRAS, 368, 1404

An T., \& Baan W. A., 2012, ApJ, 760, 77

An T., Hong, X. Y., Hardcastle M. J., et al., 2010, MNRAS, 402, 87

Antognini J., Bird J., Martini P., 2012 ApJ, 756, 116

Antoniadis J. et al., 2015, 'Multi-wavelength, Multi-Messenger Pulsar Science in the SKA Era', in proceedings of 'Advancing Astrophysics with the Square Kilometre Array', PoS(AASKA14)157 
Bacon D. et al., 2015, 'Large Synoptic Survey Telescope synergy with the Square Kilometre Array', in proceedings of 'Advancing Astrophysics with the Square Kilometre Array', PoS(AASKA14) 145

Barthel P. D., 1989, ApJ, 336, 606

Barthel P. D., \& Arnaud K. A., 1996, MNRAS, 283, L45

Baum S. A., O’Dea C. P., Murphy D. W., \& de Bruyn A. G., 1990, A\&A, 232, 19

Becker R. H., White R. L., \& Helfand D. J., 1995, ApJ, 450, 559

Best P. N., Ker L. M., Simpson C., Rigby E. E., \& Sabater, J., 2014, MNRAS, 445, 955

Best P. N., \& Heckman T. M., 2012, MNRAS, 421, 1569

Bîrzan L., Rafferty D. A., McNamara B. R., Wise M. W., \& Nulsen P. E. J., 2004, ApJ, 607, 800

Blake G. M., 1970, ApL, 6, 201

Blandford R. D., \& Rees M. J., 1974, MNRAS, 169, 395

Blundell K. M., Rawlings S., \& Willott C. J., 1999, AJ, 117, 677

Bower R. G., Benson A. J., Malbon R., et al., 2006, MNRAS, 370, 645

Brocksopp C., Kaiser C. R., Schoenmakers A. P., \& de Bruyn A. G., 2007, MNRAS, 382, 1019

Brocksopp C., Kaiser C. R., Schoenmakers A. P., \& de Bruyn A. G., 2011, MNRAS, 410, 484

Broderick J. W., \& Fender R. P., 2011, MNRAS, 417, 184

Callingham J., Gaensler B., Ekers R., et al., 2015, to be submitted

Camera S. et al., 2015, 'Cosmology on the largest scales with the SKA', in proceedings of 'Advancing Astrophysics with the Square Kilometre Array', PoS(AASKA14)025

Carvalho J. C., 1985, MNRAS, 215, 463

Cattaneo A., Faber S. M., Binney J., et al, 2009, Nature, 460, 213

Cavagnolo K. W., McNamara B. R., Nulsen P. E. J., et al., 2010, ApJ, 720, 1066

Celotti A., 2003, NewAR, 47, 525

Condon J. J., Cotton W. D., Greisen E. W., et al., 1998, AJ, 115, 1693

Cordey R. A., 1987, MNRAS, 227, 695

Croston J. H., Kraft R. P., \& Hardcastle M. J., 2007, ApJ, 660, 191

Croston J. H., Kraft R. P., Hardcastle M. J., et al., 2009, MNRAS, 395, 1999

Croton D. J., Springel. V., White S. D. M., et al., 2006, MNRAS, 365, 11

Johnston-Hollitt., M. et al., 2015, 'Using Tailed Radio Galaxies to Probe the Environment and Magnetic Field of Galaxy Clusters in the SKA Era', in proceedings of 'Advancing Astrophysics with the Square Kilometre Array', PoS(AASKA14)101

Dewdney P., Turner W., Millenaar R., et al., 2013, 'SKA1 System Baseline Design', Document no. SKA-TEL-SK0-DD-001 Revision 1

Donoso E., Best P. N., \& Kauffmann G., 2009, MNRAS, 392, 617

Dunlop J. S. \& Peacock J. A., 1990, MNRAS, 247, 19

Dwarakanath K. S., \& Kale R., 2009, ApJL, 698, L163

Falcke H., Körding E., \& Nagar N. M., 2004, NewAR, 48, 1157

Fanaroff B. L., \& Riley J. M., 1974, MNRAS, 167, 31P

Fanti R., Fanti C., Schilizzi R. T., et al., 1990, A\&A, 231, 333

Ferramacho L. D., Santos M. G., Jarvis M. J., \& Camera S., 2014, MNRAS, 442, 2511

Godfrey L. E. H., \& Shabala S. S., 2013, ApJ, 767, 12

Gopal-Krishna, \& Wiita P. J., 2000, A\&A, 363, 507 
Hardcastle M. J., Evans D. A., \& Croston J. H., 2006, MNRAS, 370, 1893

Hardcastle M. J., \& Krause M. G. H., 2013, MNRAS, 430, 174

Hardcastle M. J., \& Krause M. G. H., 2014, MNRAS, 443, 1482

Hardcastle M. J., Massaro F., Harris D. E., et al, 2012, MNRAS, 424, 1774

Harwood J. J., Hardcastle M. J., Croston J. H., \& Goodger J. L., 2013, MNRAS, 435, 3353

Heesen V., Croston J. H., Harwood J. J., Hardcastle M. J., \& Hota A., 2014, MNRAS, 439, 1364

Hurley-Walker N., Jonhston-Hollitt M., Ekers R., et al., 2015, MNRAS, accepted, arXiv:1412:3856

Ineson J., Croston J. H., Hardcastle M. J., et al., 2013, ApJ, 770, 136

Jackson C. A., \& Wall J. V., 1999, MNRAS, 304, 160

Jamrozy M., Konar C., Machalski J., \& Saikia D. J., 2008, MNRAS, 385, 1286

Jarvis M. et al., 2015, 'The star-formation history of the Universe with the SKA', in proceedings of 'Advancing Astrophysics with the Square Kilometre Array', PoS(AASKA14)068

Janssen R. M. J., Röttgering H. J. A., Best P. N., \& Brinchmann, J., 2012, A\&A, 541, 62

Kaiser C. R., \& Alexander P., 1997, MNRAS, 286, 215

Kaiser C. R., \& Cotter G., 2002, MNRAS, 336, 649

Kaiser C. R., Dennett-Thorpe J., \& Alexander P., 1997, MNRAS, 292, 723

Kaiser C. R., Schoenmakers A. P., \& Röttgering H. J. A., 2000, MNRAS, 315, 381

Kapińska A. D., Andernach H., Banfield J., et al., 2015, 'Radio Galaxy Zoo: Hybrid morphology radio galaxies', to be submitted

Kapińska A. D., \& Uttley P., 2013, AN, 334, 408

Kapińska A. D., Uttley P., \& Kaiser C. R., 2012, MNRAS, 424, 2028

Karouzos M., Jarvis M. J., \& Bonfield D., 2014, MNRAS, 439, 861

Kaviraj S., Shabala S. S., Deller A. T., \& Middelberg E., 2014, submitted, arXiv:1411.2028

Kimball A. E., Kellermann K. I., Condon J. J., Ivezic Z., \& Perley R. A., 2011, ApJ, 739, 29

King I., 1962, AJ, 67, 471

Kitching T. et al., 2015, 'Euclid-SKA Synergies', in proceedings of 'Advancing Astrophysics with the Square Kilometre Array', PoS(AASKA14)146

Komberg B. V., \& Pashchenko I. N., 2009, Astronomy Reports, 53, 1086

Komissarov S. S., \& Gubanov A. G., 1994, A\&A, 285, 27

Konar C., \& Hardcastle M. J., 2013, MNRAS, 436, 1595

Kunert-Bajraszewska M., Gawroński M. P., Labiano A., \& Siemiginowska A., 2010, MNRAS, 408, 2261

Kunert-Bajraszewska M., Marecki A., \& Spencer R. E., 2004, in European VLBI Network on New Developments in VLBI Science and Technology, Bachiller R., Colomer F., Desmurs J.-F., de Vicente P., eds., pp. 73-76

Kuźmicz A., \& Jamrozy M., 2013, Advances in Astronomy and Space Physics, 3, 42

Laing R. A., 2015, 'Kinematics and Dynamics of kiloparsec-scale Jets in Radio Galaxies with SKA', in proceedings of 'Advancing Astrophysics with the Square Kilometre Array', PoS(AASKA14) 107

Laing R. A., Canvin J. R., \& Bridle A. H., 2003, NewAR, 47, 577

Luo Q., \& Sadler E. M., 2010, ApJ, 713, 398

Maccagni F. M., Morganti R., Oosterloo T. A., \& Mahony E. K., 2014, A\&A, 571, 67

Machalski J., \& Jamrozy M., 2006, A\&A, 454, 95 
Maciel T., \& Alexander P., 2014, MNRAS, 442, 3469

Mahony E. K., Sadler E. M., Croom S. M., et al., 2012, ApJ, 754, 12

Marecki A., Spencer R. E., \& Kunert M., 2003, PASA, 20, 46

Mauch T., \& Sadler E. M., 2007, MNRAS, 375, 931

McAlpine K., Jarvis M. J., \& Bonfield D. G., 2013, MNRAS, 436, 1084

McAlpine K. et al., 2015, 'The SKA view of the Interplay between SF and AGN Activity, and its role in Galaxy Evolution', in proceedings of 'Advancing Astrophysics with the Square Kilometre Array', PoS(AASKA14)083

McNamara B. R., \& Nulsen P. E. J., 2007, ARA\&A, 45, 117

McNamara B. R., \& Nulsen P. E. J., 2012, New Journal of Physics, 14, 055023

Merloni A., \& Heinz S., 2008, MNRAS, 388, 1011

Mingo B., Hardcastle M. J., Croston J. H., et al., 2012, ApJ, 758, 95

Murgia M., Fanti C., Fanti R., et al., 1999, A\&A, 345, 769

O'Dea C. P., 1998, PASP, 110, 493

O'Dea C. P., Baum S. A., \& Stanghellini C., 1991, ApJ, 380, 66

Orienti M., D’Ammando F., Giroletti M., Giovannini G., \& Panessa F., 2015, 'The physics of the radio emission in the quiet side of the AGN population with the SKA', in proceedings of 'Advancing Astrophysics with the Square Kilometre Array', PoS(AASKA14)087

Owen F. N., Ledlow M. J., \& Keel W. C., 1996, AJ, 111, 53

Owsianik I., \& Conway J. E., 1998, A\&A, 337, 69

Parma P., Murgia M., de Ruiter H. R., et al., 2007, A\&A, 470, 875

Peacock J. A., \& Wall J. V., 1982, MNRAS, 198, 843

Perucho M., \& Martí J. M., 2007, MNRAS, 382, 526

Perucho M., Martí J. M., Laing R. A., \& Hardee P. E., 2014, MNRAS, 441, 1488

Phillips R. B., \& Mutel R. L., 1982, A\&A, 106, 21

Planck Collaboration et al., 2013, arXiv:1303.5076

Polatidis A. G., \& Conway J. E., 2003, PASA, 20, 69

Rawlings S., \& Saunders R., 1991, Nature, 349, 138

Readhead A. C. S., 1995, Proceedings of the National Academy of Science, 92, 11447

Readhead A. C. S., Taylor G. B., Pearson T. J., \& Wilkinson P. N., 1996, ApJ, 460, 634

Reynolds C. S., \& Begelman M. C., 1997, ApJL, 487, L135

Rigby E. E., Best P. N., Brookes M. H., et al., 2011, MNRAS, 416, 1900

Sadler E. M., Cannon R. D., Mauch T., et al., 2007, MNRAS, 381, 211

Saikia D. J., \& Jamrozy M., 2009, Bull. Astr. Soc. India, 37, 63

Saripalli L., 2012, AJ, 144, 85

Scheuer P. A. G., 1974, MNRAS, 166, 513

Schoenmakers A. P., de Bruyn A. G., Röttgering H. J. A., et al., 2000, MNRAS, 315, 371

Shabala S. S., Ash S., Alexander P., \& Riley J. M., 2008, MNRAS, 388, 625

Shabala S. S., \& Alexander P., 2009, ApJ, 699, 525

Shabala S. S., Kaviraj S., \& Silk J., 2011, MNRAS, 413, 2815

Simpson C., Rawlings S., Ivison R., et al., 2012, MNRAS, 421, 3060

Sirothia S. K., Gopal-Krishna, \& Wiita P. J., 2013, ApJL, 765, L11

Snellen I. A. G., Schilizzi R. T., Miley G. K., et al., 1999, NewAR, 43, 675 
Snellen I. A. G., Schilizzi R. T., Miley G. K., et al., 2000, MNRAS, 319, 445

Snellen I. A. G., Schilizzi R. T., \& van Langevelde H. J., 2000, MNRAS, 319, 429

Stanghellini C., Baum S. A., O’Dea C. P., \& Morris G. B., 1990, A\&A, 233, 379

Tschager W., Schilizzi R. T., Röttgering H. J. A., et al., 2003, A\&A, 402, 171

Turner R., \& Shabala S. S., 2015, MNRAS, submitted

Urry, C. M., \& Padovani P., 1995, PASP, 107, 803

van Breugel W., Miley G., \& Heckman T., 1984, AJ, 89, 5

Wall J. V., 1994, Australian Journal of Physics, 47, 625

Wall J. V., \& Jackson C. A., 1997, MNRAS, 290, L17

Wan L., Daly R. A., \& Guerra E. J., 2000, ApJ, 544, 671

Wang Y., Knigge C., Croston J. H., \& Pavlovski G., 2011, MNRAS, 418, 1138

Wilkinson P. N., Polatidis A. G., Readhead A. C. S., Xu W., \& Pearson T. J., 1994, ApJL, 432, L87

Willott C. J., Rawlings S., Blundell K. M., \& Lacy M., 1999, MNRAS, 309, 1017

Wilman R. J., Miller L., Jarvis M. J., et al., 2008, MNRAS, 388, 1335 